\title{
Treatment of ventilator-associated pneumonia with piperacillin-tazobactum and amikacin vs cefepime and levofloxacin: A randomized prospective study
}

\author{
S. M. Ahmed, J. Choudhary, M. Ahmed, V. Arora, Parul, S. Ali
}

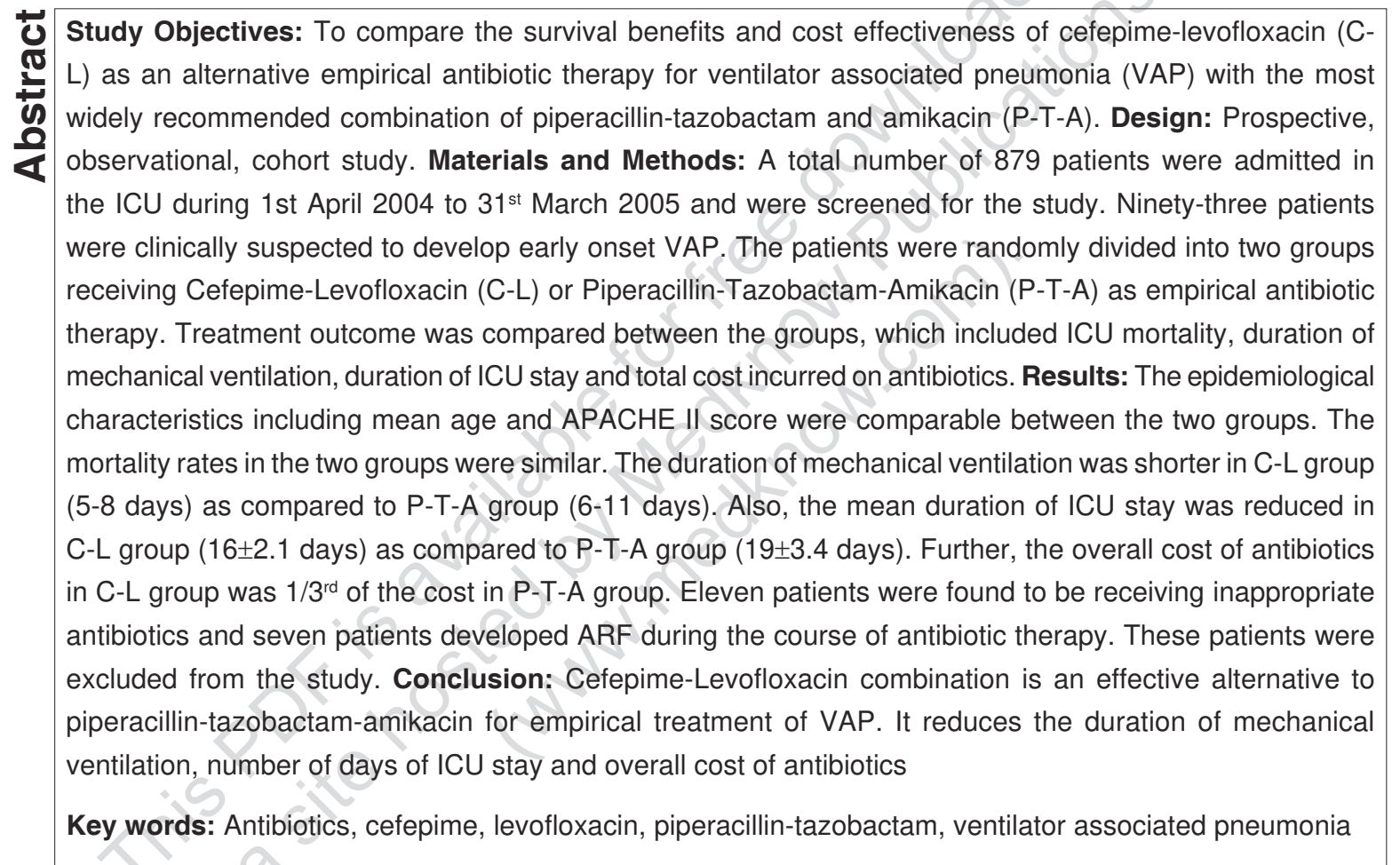

\section{Introduction}

Ventilator associated pneumonia (VAP) is associated with prolonged mechanical ventilation, increased duration of intensive care unit (ICU) stay and highest mortality

\footnotetext{
From:

Department of Anaesthesiology and Critical Care, JN Medical College, AMU, Aligarh, India

Correspondence:

Dr. S Moied Ahmed, Reader, Incharge ICU, Department of Anaesthesiology, JN Medical College, AMU, Aligarh - 202002, India.

E-mail: sma99@rediffmail.com
}

rate of all hospital acquired infections..$^{[1,2]}$ These patients are often treated empirically with antibiotic regimens based on suspected pathogens. Empirical treatment is dependent on individual patient factors and bacterial culture-sensitivity pattern of individual Intensive Care Units.

Selection of appropriate antibiotics in the initial stages is an important determinant of clinical outcome..$^{[3]}$ Various studies have shown that as much as $50 \%$ of antibiotic use is inappropriate. ${ }^{[4,5]}$ Use of appropriate antibiotics 
directed towards the most prevalent organism not only improves the cure rate and survival but also reduces the emergence of resistant strains. Most of these empirical therapies are directed towards Gram-negative aerobic bacteria and Staphylococci.

Many authorities recommend piperacillin-tazobactam as the most effective agent in the empirical treatment of VAP. ${ }^{[6]}$ However, the widespread use of piperacillintazobactam as empirical therapy has led to the development of resistance. ${ }^{[7]}$ Recently a combination of cefepime and levofloxacin has been found to be highly effective against $P$. aeruginosa, E. coli and $S$. aureus. ${ }^{[8]}$ These are found to be the most commonly isolated organisms in VAP patients.

Hence the aim of the present study was to compare the duration of mechanical ventilation, length of ICU stay, cost incurred and mortality, in patients with VAP, treated with two different groups of antibiotics (piperacillin - tazobactam and amikacin versus cefepime and levofloxacin).

\section{Materials and Methods}

The study was conducted in the Intensive Care Unit of our Institution. After obtaining approval from the institutional ethics committee, all mechanically ventilated patients who were admitted during $1^{\text {st }}$ April 2004 to $31^{\text {st }}$ March 2005 were screened and enrolled for the study.

\section{Inclusion criteria}

Patients, who were clinically suspected as a case of pneumonia after $48 \mathrm{~h}$ but within seven days of initiation of mechanical ventilation, were enrolled for the study. ${ }^{[5]}$ Clinical suspicion of VAP was defined as a new, progressive or persistent (>24 hours) infiltrate on chest radiograph, with two or more of the following criteria.

1. Fever $>38.3^{\circ} \mathrm{C}$ or hypothermia $<36^{\circ} \mathrm{C}$.

2. Purulent endotracheal aspirate.

3. Leukocyte count $>10,000 / \mathrm{mm}^{3}$ or $<4,000 / \mathrm{mm}^{3}$.

Once VAP was suspected according to the clinical criteria, the endotracheal aspirate was sent for culture and sensitivity. The existing antibiotic regimen was changed to as per the study design. The patients were randomly divided into two groups according to the empirical antibiotic regimen prescribed. All the patients enrolled with odd numbers were designated as Group C-L and received Cefepime-Levofloxacin. Patients with even numbers were designated as Group P-T-A and received Piperacillin-Tazobactam-Amikacin. The antibiotics were continued for fourteen days in both the groups.

\section{Exclusion criteria}

Patients whose culture sensitivity reports showed resistance to the prescribed antibiotics (P-T-A or C-L) were labelled as inappropriate antibiotic therapy and were excluded from the study. The number of such patients was recorded and their antibiotics were changed according to the antibiogram. Patients with known acute renal failure or chronic renal failure (ARF/CRF) were not included in the study. Patients developing ARF during the course of antibiotic therapy were also excluded from the study.

Doctors on duty made all observations and recorded relevant data from the patients' ICU records, bedside progress charts and microbiologic reports. Patient related variables that were recorded included age, sex, diagnosis, hospital and ICU admission dates. All factors necessary for calculation of APACHE II score were also recorded. Radiologists, who interpreted chest radiographs daily, were blind to the antibiotic groups. Consultants or residents on duty, who were not a part of the study, assessed the extubation criteria and the patients were extubated after they attained extubation criteria.

The treatment variables that were subsequently recorded included - i) the date VAP was first suspected; ii) whether endotracheal aspirate culture led to change of antibiotic therapy (inappropriate therapy); iii) date of liberation from mechanical ventilation; iv) date of discharge from ICU and hospital and v) date of death, if any.

The treatment outcome compared between the two groups included - i) ICU mortality, ii) duration of mechanical ventilation, iii) duration of ICU stay and iv) total cost incurred on antibiotics.

\section{Statistics}

Baseline characteristics of patients were compared with the unpaired $t$ test. Differences were considered significant if the $P$ value was below 0.05 . 


\section{Results}

Out of 879 patients admitted in the ICU during the study period, 93 patients (C-L, $n=46$ and P-T-A, $n=47$ ) met the inclusion criteria.

\section{Outcomes}

Both the groups were comparable with respect to epidemiology and clinical characteristics [Table 1].

Total observed mortality was $37.3 \%$. It was $39.68 \%$ in the Group P-T-A as compared to $35 \%$ in Group C-L. The difference in the incidence of mortality between the two groups was statistically insignificant though the rate was comparatively less in Group C-L [Table 2].

The duration of mechanical ventilation after the start of empirical therapy was $6.3 \pm 1.6$ days in the Group C$\mathrm{L}$ as compared to $8.2 \pm 2.1$ days in P-T-A Group. This difference achieved statistical significance $(P$-value $<0.05)$ [Table 3].

Further, the mean \pm SD duration of ICU stay was $16 \pm 2.1$

\begin{tabular}{lcc}
\hline $\begin{array}{l}\text { Table 1: Epidemiologic and clinical characteristics of } \\
\text { both the groups }\end{array}$ & & \\
\hline Parameters & $45.2 \pm 5.1$ & $43.6 \pm 6.2$ \\
Age (years) & $25 / 21$ & $28 / 19$ \\
Sex (M/F) & $18 \pm 2$ & $16 \pm 3$ \\
APACHE II & $5 \pm 1.4$ & $4 \pm 2.8$ \\
Duration of mechanical & & \\
ventilation before onset of VAP & & \\
Indications for mechanical ventilation & 18 & 20 \\
$\quad$ Exacerbation of COPD & 11 & 8 \\
$\quad$ Abdominal sepsis & 2 & 1 \\
Organophosphate poisoning & 3 & 5 \\
Snake bite & 5 & 6 \\
Emergency abdominal surgery & & \\
(Post-operative) & 4 & 2 \\
Pneumonia & 4 & 4 \\
Trauma & 47 & 46 \\
Total & & \\
VAP - Ventilator associated pneumonia, COPD - Chronic obstructive \\
pulmonary diseases
\end{tabular}

Table 2: Characteristics of patients included in the study cohort

\begin{tabular}{lcc}
\hline Parameters & C-L & P-T-A \\
No. of patients with clinically suspected VAP & 47 & 46 \\
Inappropriate therapy & 5 & 3 \\
Patients excluded because of acute renal failure & 4 & 5 \\
Average duration of mechanical ventilation & & \\
after suspicion of VAP (days) & $6.3 \pm 1.6$ & $8.2 \pm 2.1$ \\
Average duration of ICU stay (days) & $16 \pm 2.1$ & $19 \pm 3.4$ \\
Incidence of death & $35 \%$ & $39.68 \%$ \\
\hline
\end{tabular}

VAP - Ventilator associated pneumonia, ICU - Intensive care unit days in CL Group as compared to $19 \pm 3.4$ days in PTA Group. The difference was statistically significant $(P$ value<0.05) [Table 3]

Eight patients were found to be receiving inappropriate antibiotics, as the organism isolated from their endotracheal aspirate was resistant to the empirical regimen used. Nine patients in the study cohort developed ARF during the course of therapy and had to be excluded from the study [Table 2].

Patient factors such as high APACHE II score on admission, immunocompromised state and hepatic failure were associated with increased mortality in both the groups [Table 4].

\section{Pathogens}

Endotracheal aspirate was obtained from all patients. The most commonly isolated organisms were $P$. aeruginosa, Staphylococcus aureus and E. coli [Table 3].

\section{Cost effectiveness}

As evident in [Table 5], C-L combination is more economical than P-T-A. The average cost of antibiotics

Table 3: Organisms isolated from clinically suspected ventilator associated pneumonia patients in both the groups

\begin{tabular}{lcc}
\hline Organisms & $\begin{array}{c}\text { Group C-L } \\
(\mathbf{n}=\mathbf{4 7})\end{array}$ & $\begin{array}{c}\text { Group P-T-A } \\
(\mathbf{n}=\mathbf{4 6})\end{array}$ \\
P. aeruginosa & 20 & 17 \\
Staphylococcus aureus & 11 & 14 \\
E. coli & 7 & 10 \\
Acinetobacter & 3 & 2 \\
Klebsiella & 2 & 2 \\
Streptococcus species & 3 & 2 \\
\hline
\end{tabular}

Table 4: Patient factors associated with high mortality in both the groups

\begin{tabular}{lccccc}
\hline & \multicolumn{2}{c}{ C-L } & & \multicolumn{2}{c}{ P-T-A } \\
\cline { 2 - 3 } $\begin{array}{l}\text { No. of } \\
\text { patients }\end{array}$ & Mortality & & $\begin{array}{c}\text { No. of } \\
\text { patients }\end{array}$ & Mortality \\
$\begin{array}{l}\text { Hepatic failure } \\
\text { Immunocompromised }\end{array}$ & 6 & 4 & & 5 & 4 \\
state & 3 & 2 & & 3 & 2 \\
Apache II $>20$ & 2 & 1 & & 2 & 1 \\
\hline
\end{tabular}

Table 5: Comparison of average cost of antibiotic in both the groups

\begin{tabular}{lcc}
\hline Average cost of antibiotics (Rs.) & C-L & P-T-A \\
Per day & 554.30 & 1689.80 \\
Per patient & 7760.20 & 23657.20 \\
\hline
\end{tabular}


incurred in C-L group was Rs. 554.30 per day per patient as compared to Rs. 1689.80 in the P-T-A group. Hence, the cost incurred in $C-L$ group was approximately $1 / 3^{\text {rd }}$ to that of P-T-A group.

\section{Discussion}

Antibiotics are one of the costliest categories of drugs used in the ICU. Its use has become mandatory as it influences the patient outcome in the ICU. Patient outcome also depends upon early, appropriate and adequate administration of empirical antibiotics. ${ }^{[6,9]}$ However, there are no specific guidelines regarding empirical antibiotic treatment of VAP. ${ }^{[6]}$ Initially various authorities investigated single drug therapy as the empirical treatment of VAP. ${ }^{[10,11]}$ Because of development of resistance and high mortality associated with monotherapy, use of two antibiotics directed against most commonly isolated organisms is recommended now. ${ }^{[12,13]}$ Recently, the most frequently recommended combination therapy includes antipseudomonal penicillin plus betalactamase inhibitor along with fluoroquinolones or aminoglycosides. ${ }^{[6]}$

It is very difficult to conduct a study free from bias. However, in our study we took certain steps to make it bias free. The patients fulfilling the clinical criteria of VAP were randomly divided into two groups. VAP criteria were applied by consultants who were not a part of the study. Further, patients in both the groups were taken off the ventilator when they met a well-defined extubation criteria assessed by consultants or residents who were independent. Even the radiologist commenting on chest radiographs was made blind to the groups.

In the present study the survival outcome was similar in both the groups. However, patients treated with cefepime plus levofloxacin had shorter duration of mechanical ventilation and ICU stay as compared to piperacillin-tazobactam-amikacin combination. This may be attributed to rapid, synergistic bactericidal activity of cefepime - levofloxacin combination. ${ }^{[8]}$ Further, since tazobactam is a suicidal antibiotic it can develop resistance in the parent antibiotic. The organisms isolated in our ICU might be developing resistance in vivo to this combination, which was not evident in the in vitro sensitivity investigations. As an advantage over these suicidal antibiotics, cefepime - levofloxacin combination has been shown to slow and prevent the development of resistance. ${ }^{[8]}$ Contrary to our observations, other studies conducted with fluoroquinolones and cephalosporins did not show any improvement in the outcome in VAP patients. ${ }^{[6]}$ This could be because the previous studies have compared second and third generation cephalosporin. Cefepime, a fourth generation cephalosporin has a structural advantage (Zwitterionic configuration) which allows faster penetration through the cell membrane of gram negative bacteria and makes it more stable against beta lactamases as compared to third generation cephalosporins. ${ }^{[14]}$

Cefepime-levofloxacin combination reduced the total cost of antibiotics to nearly $1 / 3$ of the cost of piperacillintazobactam-amikacin combination [Table 5]. Reduced number of days of mechanical ventilation and ICU stay further made cefepime-levofloxacin combination a cost effective alternative to piperacillin-tazobactam-amikacin [Table 2].

In our study there was no recurrence of pneumonia after the completion of antibiotic treatment. We discharged the patients once they were symptom free.

Four patients in the cefepime-levofloxacin group and seven in the piperacillin-tazobactam-amikacin group were excluded from the study. These patients were found to be receiving inappropriate antibiotics, i.e. the isolated strains in these patients were resistant to the empirical therapy.

The patients who developed acute renal failure during the course of therapy were excluded from both the groups. They were excluded because either the dose of amikacin had to be reduced or it had to be completely avoided depending upon the level of serum creatinine. Inclusion of such patients in the cefepime-levofloxacin group would make the two groups incomparable. Further, acute renal failure is an independent risk factor for poor outcome. ${ }^{[15,16]}$

The organisms isolated in our study were similar to other study groups. ${ }^{[6]}$ Diagnostic criteria of VAP and extubation criteria were similar to that used in most ICUs. ${ }^{[6]}$ Patient factors such as immunocompromised state, hepatic failure, ARDS and higher APACHE II scores resulted in higher mortalities [Table 4]. It is in accordance with the clinical outcomes seen in other studies. ${ }^{[6,15,16]}$ 
The major limitation of our study was that it did not investigate the causative factors associated with early onset VAP. Our study was designed to investigate only the treatment outcome in two different antibiotic groups. Also we did not analyze the number of days required to make the culture negative for previously isolated organisms.

\section{Conclusion}

The results of this study indicate that combination of cefepime-levofloxacin is an effective alternative to piperacillin-tazobactam-amikacin, which is a widely accepted antibiotic regimen for the treatment of VAP. Further, cefepime-levofloxacin combination reduced the duration of mechanical ventilation, length of ICU stay, mortality and the over all cost of antibiotic therapy. However a study should be designed to evaluate the effect of these drugs in patients with ARF. Further, it should study the number of days required to make the patients culture negative with either of these combinations, which was one of the limitation of our study.

\section{References}

1. Papazian L, Bregeon F, Thirion X, Gregoire R, Saux P, Denis JP, et al. Effect of ventilator-associated pneumonia on mortality and morbidity. Am J Respir Crit Care Med 1996;154:91-7.

2. Rello J, Quintana E, Ausina V, Castella J, Luquin M, Net A, et al. Incidence, etiology and outcome of nosocomial pneumonia in mechanically ventilated patients. Chest 1991;100:439-44.

3. Alvarez-Lerma F. Modification of empiric antibiotic treatment in patients with pneumonia acquired in the intensive care unit: ICU-Acquired Pneumonia study group. Intensive Care Med 1996;22:387-94.

4. Dunagan, WC, Woodward RS, Medoff G, Gray JL, Casabar $\mathrm{E}$, Lawrenz C, et al. Antibiotic misuse in two clinical situations: Positive blood cultures and administration of aminoglycoside. Rev Infect Dis 1991;13:405-12.

5. Gruson D, Hilbert G, Vargas F, Valentino R, Bebear C, Allery A, et al. Rotation and restricted use of antibiotics in a Medical Intensive care unit. Am J Respir Crit Care Med 2000;162:837-43.

6. Fowler RA, Flavin KE, Barr J, Weinacker AB, Parsonnet J, Gould
MK. Variability in antibiotic prescribing patterns and outcomes in patients with clinically suspected ventilator-associated pneumonia. Chest 2003;123:835-44.

7. Mathai D, Rhomberg PR, Biedenbach DJ, Jones RN; India Antimicrobial Resistance Study Group. Evaluation of the in vitro activity of six broad-spectrum $\beta$-lactam antimicrobial gents tested against recent clinical isolates from India: A survey of ten medical center laboratories. Diag Microbiol Infect Dis 2002;44:367-77.

8. Fish DN, Choi MK, Jung R. Synergic activity of cephalosporins plus fluoroquinolones against Pseudomonas aeruginosa with resistance to one or both drugs. J Antimicrob Chemother 2002;50:1045-9.

9. Kollef $\mathrm{MH}$, Sherman $\mathrm{G}$, Ward S, Fraser VJ. Inadequate antimicrobial treatment of infections: A risk factor for hospital mortality among critically ill patients. Chest 1999;115:462-74.

10. Fink MP, Snydman DR, Niederman MS, Leeper KV, Johnson RH, Heard SO, et al. Treatment of severe Pneumonia in hospitalized patients: Results of multicenter, randomized, double-blind trial comparing intravenous ciprofloxacin with imipenem-cilastin. Antimicrob Agents Chemother 1994;38:547-57.

11. Leibovici L, Paul M, Poznanski O, Drucker M, Samra Z, Konigsberger $\mathrm{H}$, et al. Monotherapy vs $\beta$-lactam-aminoglycoside combination treatment for Gram-negative bacteremia: A prospective, observational study. Antimicrob Agents Chemother 1997;41:1127-33.

12. Hospital-acquired pneumonia in adults: Diagnosis, assessment of severity, initial antimicrobial therapy, and preventive strategies. A consensus statement, American Thoracic Society, November 1995. Am J Respir Crit Care Med 1995;153:1711-25.

13. Lynch JP. Hospital-acquired pneumonia: Risk-factors, microbiology and treatment. Chest 2001;119:373S-84S.

14. Kessler RE. Cefepime microbiologic profile and update. Pediatr Infect Dis J 2001;20:331-6.

15. Cook DJ, Walter SD, Cook RJ, Griffith LE, Guyatt GH, Leasa D, et al. Incidence and risk factors for ventilator associated pneumonia in critically ill patients. Ann Intern Med 1998;129:433-40.

16. Cook DJ, Kollef MH. Risk factors for ICU-acquired pneumonia. JAMA 1998;279:1605-6.

Source of Support: Nil, Conflict of Interest: None declared. 